\title{
End of the line?
}

\author{
The Higgs boson is running out of places to hide.
}

The Higgs boson, as part of the standard model, could be facing exclusion. The latest results from CERN's Large Hadron Collider (LHC), announced in Paris in November, leave only a small mass window, from 114 to $141 \mathrm{GeV}$, in which the particle might lurk. By the end of December, that window may be gone completely.

Following the shutdown earlier this year of the Tevatron at Fermilab, the two general-purpose detectors at the LHC ATLAS and CMS - now lead the way in the search for the elusive boson. At the summer conferences, both collaborations showed results that contained a tentative hint of something unusual - a slight excess of events - around the $140 \mathrm{GeV}$ mass region. But in subsequent analyses with more data, that hint did not develop into anything more solid. Now, with the LHC approaching its winter shutdown before resuming operation early in 2012, both groups are working to extract as much as possible from the entire dataset accumulated so far. By the time of the last major event on CERN's calendar this year - the open session of its governing council on 14 December - we may know whether or not reports of the Higgs' demise have, shall we say, been exaggerated.

The Higgs mechanism has long been the leading contender to explain, in the context of the standard model of particle physics, why particles have the masses they do: the breaking of electroweak symmetry that it entails causes the weak-force carriers (the $W$ and $Z$ bosons) to have masses that are around 100 times greater than that of the proton, while the photon of electromagnetism remains massless.

So far, the data from CERN's Large Electron-Positron Collider (which closed in 2000) exclude - at 95\% confidence level - the existence of a standard-model Higgs up to $114 \mathrm{GeV}$; LHC data exclude the mass range above $141 \mathrm{GeV}$. The Tevatron's two experiments - CDF and D0 - have also excluded ranges either side of this mass window, and, although data-taking has stopped, analysis continues in these collaborations. And Tevatron data could well prove a vital part of the story, as the unexcluded mass region is particularly challenging for the LHC experiments to explore: for a Higgs mass upwards of about $114 \mathrm{GeV}$, the typical signature of a Higgs decay into a pair of bottom quarks is easier (although not easy) to spot in Tevatron data; a decay into a pair of photons is a clean signature that the LHC detectors can pick up, but the expected rate is much lower.
Over the next year, the combination of data from the four experiments - CDF, D0, ATLAS and CMS - (expected to amount to around 10 inverse femtobarns each) should reach sufficient sensitivity to firmly rule out, or rule in, the existence of the standard-model Higgs. However, the standard-model Higgs is not the only game in town: extensions to the standard model are envisaged that predict, in particle-physics speak, an extended Higgs sector. This brings the possibility of several Higgs-like particles, and these may take longer to find. Besides, if something Higgs-like does turn up in LHC data, some considerable further investigation would be necessary to ascertain exactly what it is, standard or otherwise. This is built in to the LHC programme, which will see over the next decade and more a sequence of data-taking and of collider and experiment upgrades.

These are exciting times. The Higgs mechanism, postulated in the 1960s, is facing its most stringent test. Whether or not it is proved in the short term, the LHC promises a long-term definitive investigation. There are theorists who would relish a nondiscovery, for the back-to-the-drawingboard rethink of particle physics that it would entail. However, whatever is found or not found at the LHC, let there at least be a clear signal of the way forward.

\section{Hard times}

\section{Investment in science must be part of Europe's plan to rebuild its economies.}

Europe is embroiled in a major crisis. One by one, Eurozone countries have been toppling into unsustainable debt. The continent is expected to face serious recession during 2012, as the ability of economically stronger Eurozone members to bail out the weaker is exhausted.

Following Greece, then Italy, Spain has become the latest focus of concern and also the latest nation to face a radical change in government. In both Greece and Italy, technocratic administrations unelected - are now in place. Spain's governmental overhaul, however, came at the hands of its electorate, when opposition conservatives won a landslide victory in November. And, in further contrast to the technocrats of Greece and Italy who have promised to move fast to alleviate their countries' economic woes, Spain's new administration does not, by law, take office until late in December.

So it remains to be seen what will become of Spain's Severo-Ochoa scheme, established by the outgoing administration expressly to preserve excellence in its science programme in the face of economic difficulties. Eight research centres, including the Barcelona Supercomputing Centre and the Instituto de Ciencias Fotónicas, have so far won funding of $€ 5$ million over the next five years.
According to the 2010 Science, Technology and Industry Outlook produced by the Organisation for Economic Co-operation and Development (of which 34 countries are members), Spain and Greece produce a percentage of science and engineering degrees relative to all degrees that is higher than the OECD average; Italy's percentage matches the average. Active government strategy for the advancement of science, despite economic challenges, was also noted for Spain and Greece.

The future certainly isn't bright, anywhere in Europe. But recognition of the role of science and innovation in economic recovery - and acting to maintain it is vital. 